\title{
A manual way to the ancestral genome
}

Characterizing the ancestral genome of a species is of great evolutionary interest. Computational techniques have already been used to reconstruct ancestral genomes of several species, but these methods have limitations - in one algorithm, for example, only one species or a few outgroups can be compared at a time. In a recent paper, Gordon et al. use a manual, parsimony-based approach to identify the gene content and order of an extinct ancestral yeast species just before the whole genome duplication (WGD) event $\sim 100$ million years ago in the lineage leading to Saccharomyces cerevisiae and related species. This

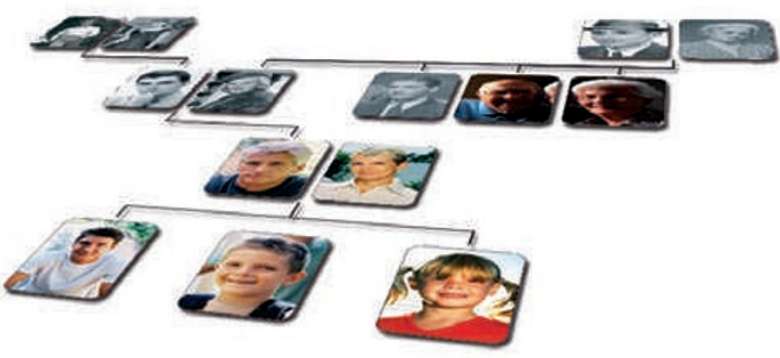

reconstruction is being used as the 'gold standard' against which to test computational methods.

The authors compared genome data from the Yeast Gene Order Browser (YGOB) of five post-WGD yeast species (descended from the ancestor that underwent WGD) and six non-WGD yeast species. They visually compared overlapping 25 -gene windows across the genomes to find breaks in synteny and deduce the ancestral gene order.

Their method inferred an ancestral genome with 8 chromosomes and 4,703 protein-coding genes, which can be viewed on the YGOB website. The authors acknowledge that their method cannot cover telomeric regions, genes that were transposed at approximately the same time as the WGD or genes that were lost in all post-WGD species after the WGD event.

In spite of these limitations, the manual reconstruction has inferred an ancestral yeast genome that is more complete than those obtained by computational approaches. By comparing the ancestral genome with a current genome as well as examining the structural rearrangements and gene gains and losses that occurred since the WGD, the evolutionary pressures that have shaped the genome can be identified. This can give insight into how species adapt to changes in the environment and the mechanisms that underlie genomic changes. As more yeast genomes are sequenced, the new data can be incorporated into the manual reconstruction, bringing us closer to a complete ancestral yeast genome.

Elizabeth Neame

ORIGINAL RESEARCH PAPER Gordon, J. L., Byrne, K. P. \& Wolfe, K. H. Additions, losses, and rearrangements on the evolutionary route from a reconstructed ancestor to the modern

Saccharomyces cerevisiae genome. PLoS Genet. 5 e1000485 (2009)

FURTHER READING Conant, G. C. \& Wolfe, K. H. Turning a hobby into a job: how duplicated genes find new functions. Nature Rev. Genet. 9, 938-950 (2008)

WEBSITE

Yeast Gene Order Browser:

http://wolfe.gen.tcd.ie/ygob 